\title{
Mitochondria-targeted antioxidant therapy for an animal model of PCOS-IR
}

\author{
YU DING $^{1 *}$, ZHAOCHANG JIANG $^{2 *}$, BOHOU XIA $^{3}$, LIZONG ZHANG $^{4}$, CAIJUAN ZHANG ${ }^{5}$ and JIANHANG LENG ${ }^{1}$ \\ ${ }^{1}$ Central Laboratory, Hangzhou First People's Hospital; ${ }^{2}$ Department of Pathology, Second Affiliated Hospital, \\ Zhejiang University, School of Medicine, Hangzhou, Zhejiang 310006; ${ }^{3}$ Department of Pharmacy, \\ Hunan Chinese Medical University, Changsha, Hunan 410208; ${ }^{4}$ Department of Experimental Animal Center, \\ Zhejiang Chinese Medical University, Hangzhou, Zhejiang 310053; ${ }^{5}$ Department of Gynecological and Obstetrics, \\ Hangzhou First People's Hospital, Zhejiang University, School of Medicine, Hangzhou, Zhejiang 310006, P.R. China
}

Received June 16, 2018; Accepted October 19, 2018

DOI: $10.3892 /$ ijmm.2018.3977

\begin{abstract}
Polycystic ovary syndrome (PCOS) is a common endocrine disorder with unknown etiology and unsatisfactory clinical treatment. Considering the ethical limitations of studies involving humans, animal models that reflect features of PCOS and insulin resistance (IR) are crucial resources in investigating this syndrome. Our previous study showed that mitochondrial dysfunction resulted from pathogenic mutations of mitochondrial DNA (mtDNA), and that oxidative stress had an active role in the phenotypic manifestation of PCOS-IR. Therefore, it was hypothesized that limiting oxidative stress and mitochondrial damage may be useful and effective for the clinical treatment of PCOS-IR. For this purpose, the present study examined the therapeutic effects of the mitochondria-targeted antioxidant $\mathrm{Mito}_{10}$ for PCOS-IR. Furthermore, the histopathology was used to analysis the ovarian morphological changes. The endocrine and reproductive related parameters were analyzed by ELISA approach. A PCOS-IR model was successfully established by subcutaneous injection of rats with testosterone propionate and feeding a high-fat diet. The 30 female Sprague-Dawley rats were then divided into three groups, comprising a control $(\mathrm{n}=10)$, animal model (PCOS-IR, $\mathrm{n}=10)$ and $\mathrm{Mito}_{10}$ treatment $(n=10)$ group. It was found that $\mathrm{Mito}_{10}$ significantly improved the IR condition and reversed the endocrine and reproductive conditions of PCOS. In addition, the impaired mitochondrial functions were improved following Mito $_{10}$ administration. Notably, western blot results suggested that this antioxidant
\end{abstract}

Correspondence to: Dr Yu Ding, Central Laboratory, Hangzhou First People's Hospital, Zhejiang University, School of Medicine, 261 Huansha Road, Hangzhou, Zhejiang 310006, P.R. China

E-mail: dingyu.zj@gmail.com

*Contributed equally

Key words: mitochondrial, MitoQ $_{10}$, antioxidant therapy, animal model, polycystic ovary syndrome and insulin resistance reduced the expression levels of apoptosis-related proteins cytochrome $c$ and B-cell lymphoma-2 (Bcl-2)-associated X protein, whereas the anti-apoptotic protein Bcl-extra large was increased following $\mathrm{Mito}_{10}$ treatment. Taken together, the data indicated that the $\mathrm{Mito}_{10}$ may have a beneficial favorable therapeutic effect on animals with PCOS-IR, most likely via the protection of mitochondrial functions and regulation of programmed cell death-related proteins.

\section{Introduction}

PCOS is a common endocrine and metabolic disease affecting $\sim 10 \%$ of women of reproductive age (1). Clinically, PCOS may manifest a variety of phenotypes, including chronic anovulation, hyperandrogenism, insulin resistance (IR) and infertility. Women with PCOS are also more prone to developing diabetes, coronary heart disease and metabolic syndrome (2). Therefore, the management of PCOS-IR remains a significant clinical challenge in gynecology.

Oxidative stress (OS), resulting from an imbalance between radicals and antioxidant defense, has been found to be a main pathophysiological mechanism in various human diseases (3). This occurs due to the overproduction of specific molecules (4), including reactive oxygen species (ROS), which are generated from nitric oxide (NO) and malondialdehyde (MDA) and can damage all components of the cell, including proteins, lipids and DNA (5). Increasing evidence indicates that OS may be associated with IR and obesity, and may also contribute to PCOS and its metabolic associations $(6,7)$.

Mitochondria produce the majority of the body's cellular energy via oxidative phosphorylation, releasing ROS as a toxic byproduct (8). The increased ROS production may have negative consequences; for example, enhancing the OS in tissues and organisms, disrupting the natural genetic code and inducing the mitochondrial-mediated apoptosis (9). Our previous studies indicated that mtDNA pathogenic mutations led to mitochondrial dysfunctions and, together with OS, contributed to the progression of PCOS-IR $(10,11)$. Considering the importance of mitochondria in energy production and ROS generation, it was hypothesized that mitochondria-targeted 
antioxidants may offer potential as a novel, potential useful drug to prevent the pathogenesis of PCOS-IR.

Mito $_{10}$ is one of these mitochondria-targeted antioxidants, which directly binds to human mitochondria. At the molecular level, it consists of a triphenylphosphonium cation $\left(\mathrm{TPP}^{+}\right)^{2}$ attached to a ubiquinone moiety (Fig. 1) (12). Previous studies have suggested that MitoQ $_{10}$ can accelerate the absorbance of $\mathrm{CoQ}_{10}$ into mitochondria in a mouse model of Alzheimer's disease, and this antioxidant is considered to combat ROS generation in mitochondria $(13,14)$. In vitro and in vivo experiments have indicated that $\mathrm{Mito}_{10}$ has antioxidant effects (13-15). In addition, Mito $_{10}$ had been found to be a novel therapeutic intervention in human autoimmune disease (15) and cardiovascular disease $(16,17)$. However, whether Mito $Q_{10}$ has a therapeutic effect on PCOS-IR remains to be elucidated.

In the present study, a rat model manifesting the features of PCOS-IR was first generated; this was followed by investigation of the effects of $\mathrm{Mito}_{10}$ on the rats and further discussion of the possible molecular mechanism.

\section{Materials and methods}

Animals. A total of 30 female Sprague-Dawley rats (SPF grade, 3 weeks old, 230-250 g) [SCXK (Hu) 2013-0016] were provided by the Experimental Animal Center, Zhejiang Chinese Medical University (Hangzhou, China). The rats were housed with four to six animals per cage under standard laboratory conditions $\left(25^{\circ} \mathrm{C}\right.$ clean environment with $50 \%$ humidity, 12-h light/dark cycle), all animals had free access to regular food and tap water. All experiments were performed in strict accordance with the Animal Care and Use Committee of Zhejiang Chinese Medical University.

Generation of the PCOS-IR rat model. The 30 rats were divided into three groups for the following stage of treatment, which were as follows: i) Control group $(n=10)$ : Mice received a normal rodent diet and injection with olive oil at a volume equal to injections in the experimental groups; ii) PCOS-IR model group $(\mathrm{n}=10): 100 \mu \mathrm{g}$ of testosterone propionate (Sigma-Aldrich; Merck KGaA, Darmstadt, Germany) was subcutaneously injected into animals, which was dissolved in $0.05 \mathrm{ml}$ commercial corn oil. The rats were also fed a high-fat diet, consisting of $54.2 \%$ standard diet, $16.8 \%$ lard, $15 \%$ sucrose, $9 \%$ casein, $1 \%$ minerals, $1 \%$ vitamins and $3 \%$ malt dextrin. Following 8 consecutive weeks of treatment, the mice were sacrificed and the vaginal cytology was analyzed using the methylene blue staining method by LEICA DM1000 light microscope (Leica Microsystems GmbH, Wetzlar, Germany); iii) Mito $_{10}$ treatment group $(n=10)$ : Following establishment of the PCOS-IR animal model, 10 PCOS-IR rats were given clean drinking water which contained $500 \mu \mathrm{mol} / 1 \mathrm{Mito}_{10}$ (Sigma-Aldrich; Merck KGaA) for 8 consecutive weeks. The solutions for $\mathrm{Mito}_{10}$ were reformulated fresh every 3 days, and were stored at $4^{\circ} \mathrm{C}$ in a dark room.

Detection of endocrine-related parameters. The orbital venous blood of each animal in the control, PCOS-IR and MitoQ treatment groups were collected following overnight fasting. The serum levels of fasting plasma glucose (FPG) and fasting
A<smiles>COC1=C(OC)C(=O)C(CCCCCCCCCC[Pb](Br)Br)=C(C)C1=O</smiles>

B

Mitochondrially targeted antioxidants

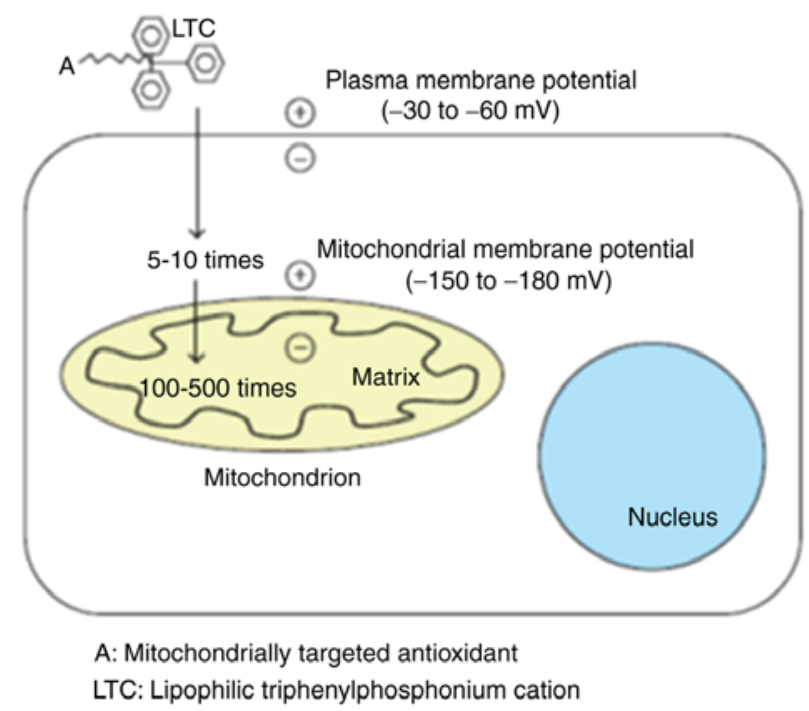

Figure 1. Structure and accumulation of $\mathrm{Mito}_{10}$. (A) Chemical structure of $\mathrm{Mito}_{10}$. (B) $\mathrm{Mito}_{10}$ accumulates (5-10 fold) within the cell driven by the plasma membrane potential, and accumulates (100-500 fold) within the mitochondria, driven by the mitochondrial membrane potential.

insulin (FINS) were then analyzed. In addition, the levels of hormones in the rats, including testosterone (T), lactate dehydrogenase ( $\mathrm{LH})$, follicle-stimulating hormone (FSH), FPG and FINS, were determined using ELISA kits (Elabscience, Wuhan, China), according to the protocols provided by the manufacturer. A total of three samples were selected from each group for analysis. The HOMA-IR was determined as follows: HOMA-IR = FPG $(\mathrm{mmol} / \mathrm{l})$ x FINS $(\mathrm{mU} / \mathrm{l}) / 22.5$; IR was considered present if the value of HOMA-IR was $>2.8$ (18).

Histopathology. All rats were bilaterally ovariectomized following treatment. The animals were anesthetized with isoflurane, and the ovaries were removed through dorsolateral incisions. The ovaries were subsequently underwent $10 \%$ formalin-fixed and paraffin-embedded, incubated overnight at $4^{\circ} \mathrm{C}$, stained with hematoxylin for $5 \mathrm{~min}$ and eosin for $3 \mathrm{~min}$ under room temperature $\left(25^{\circ} \mathrm{C}\right)$; two pathologists evaluated the morphological changes between different groups using the LEICA DM1000 light microscope (Leica Microsystems $\mathrm{GmbH})$.

Measurement of the levels of OS-associated biomarkers. To examine the expression levels of OS-related biomarkers, the contents of MDA, total antioxidant capacity (T-AOC), and the activity of superoxide dismutase (SOD) and glutathione (GSH) in tissues were analyzed using the kits provided by Nanjing Jiancheng Bioengineering Institute (Nanjing, China) according to the manufacturer's protocol. 


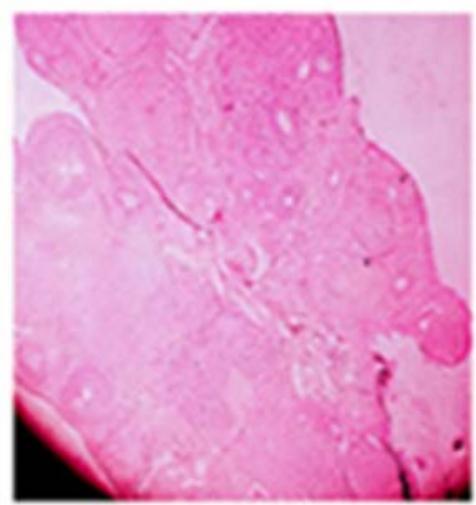

Control

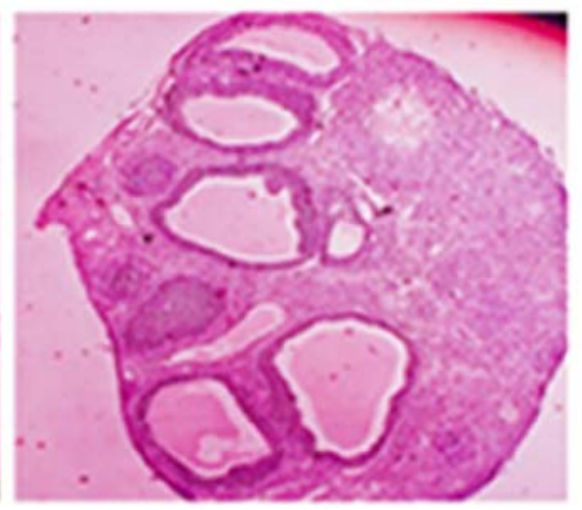

PCOS-IR

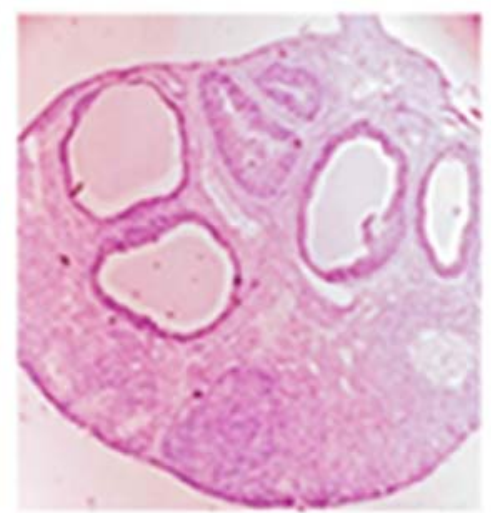

Mito $Q_{10}$ treatment

Figure 2. Features of the PCOS-IR rat model. MitoQ ${ }_{10}$ improved the morphology of ovarian follicles and increases the granulosa cells of the PCOS-IR rat model. Magnification, x200. PCOS-IR, polycystic ovary syndrome and insulin resistance.
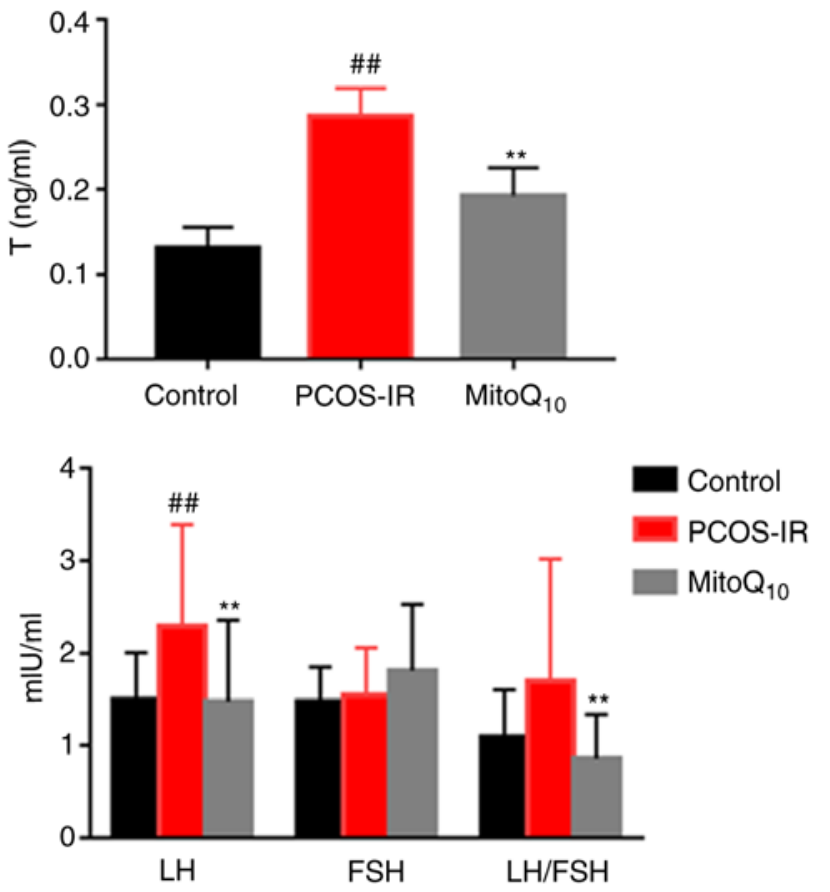

Figure 3. Serum levels of T, LH, FSH and LH/FSH in rats in the Control, PCOS-IR and Mito $_{10}$ treatment groups. ${ }^{\# \#} \mathrm{P}<0.05$, compared with the control; ${ }^{* *} \mathrm{P}<0.05$, compared with the PCOS-IR group. PCOS-IR, polycystic ovary syndrome and insulin resistance; T, testosterone; LH, lactate dehydrogenase; FSH, follicle-stimulating hormone.

ATP analysis. The ATP concentrations in the ovarian tissues were analyzed using the ATP assay kit, according to the manufacturer's protocols. The absorbance was then measured at $636 \mathrm{~nm}$ (Nanjing Jiancheng Bioengineering Institute).

Isolation of mitochondria. The isolation of mitochondria was performed at $4^{\circ} \mathrm{C}$ by different centrifugation (700 x g for $10 \mathrm{~min} ; 3,000 \mathrm{x} \mathrm{g}$ for $15 \mathrm{~min}$ ) steps using a mitochondria isolation kit (Baosai Biosciences, Inc., Beijing, China). The final pellet of mitochondria was resuspended in buffer, stored on ice, stored for $4^{\circ} \mathrm{C}$ and used for experiments within $4 \mathrm{~h}$ (19). The BCA assay was used to determine the final protein concentration.
Analysis of mitochondrial membrane potential (MMP) and ROS. MMP was determined using the JC-1 kit (cat. no. T4069, Sigma-Aldrich; Merck KGaA), according to our previous study (11). In brief, $50 \mu \mathrm{g}$ of the purified rat mitochondria protein was mixed with $5 \mu \mathrm{g} / \mathrm{ml} \mathrm{JC}-1$ staining for $10 \mathrm{~min}$ at $37^{\circ} \mathrm{C}$; subsequently, a microscope reader (Tecan Group, Inc., Ltd., Mannendorf, Switzerland) was used to record the green and red fluorescence of JC-1 at 530 and $590 \mathrm{~nm}$, respectively (20). Qualification of the ROS levels were assessed using $20 \mu \mathrm{M}$ of ROS-sensitive dye 2',7'-dichlorodihydrofluorescein diacetate $\left(\mathrm{H}_{2} \mathrm{DCFDA}\right)$, which cleaves to become DCF (cat. no. D6883; Sigma-Aldrich; Merck $\mathrm{KGaA})(21)$.

Western blot analysis. For western blot analysis, the total proteins from the fresh rat ovarian tissues were extracted based on the protocol originally proposed by Dignam et al (22) with modifications. The $50 \mu \mathrm{g}$ protein samples were boiled for 5 min in Laemmli buffer and then separated by $12 \%$ SDS polyacrylamide gel at $180 \mathrm{~V}$. Subsequently, the separated proteins were transferred onto a polyvinylidene difluoride membrane at $400 \mathrm{~mA}$ for $2 \mathrm{~h}$ (EMD Millipore, Billerica, MA, USA). Following incubation of the PVDF membrane with blocking solution, the protein bands were mixed with special anti-Bax (cat. no. 34260; 1:500; Signalway Antibody LLC, College Park, MD, USA), anti-Bcl-xL (cat. no. 21061; 1:500; Signalway Antibody LLC), anti-Cyto C (cat. no. ab33484; 1:500; Abcam) and anti-Bcl-2 (cat. no. ab59348; 1:500; Abcam) antibodies overnight at $4^{\circ} \mathrm{C}$. The blots were then washed and incubated with horseradish peroxidase (HRP)-conjugated secondary antibodies for $1 \mathrm{~h}$ at room temperature, the second antibodies were as follows: Anti-rabbit IgG, HRP-linked antibody (cat. no. 7074; 1;500; Signalway Antibody LLC) and anti-mouse IgG, HRP-linked antibody (cat. no. 7076; 1:500; Signalway Antibody LLC). The immune complexes were visualized using an enhanced chemiluminescence kit and analyzed with the Image ${ }^{\circledR}$ LAS 4000 mini program (National Institutes of Health, Bethesda, MD, USA).

Statistical analysis. All values are expressed as the mean \pm standard deviation. The statistical analyses were performed using SPSS 19.0 software (IBM SPSS, Armonk, 

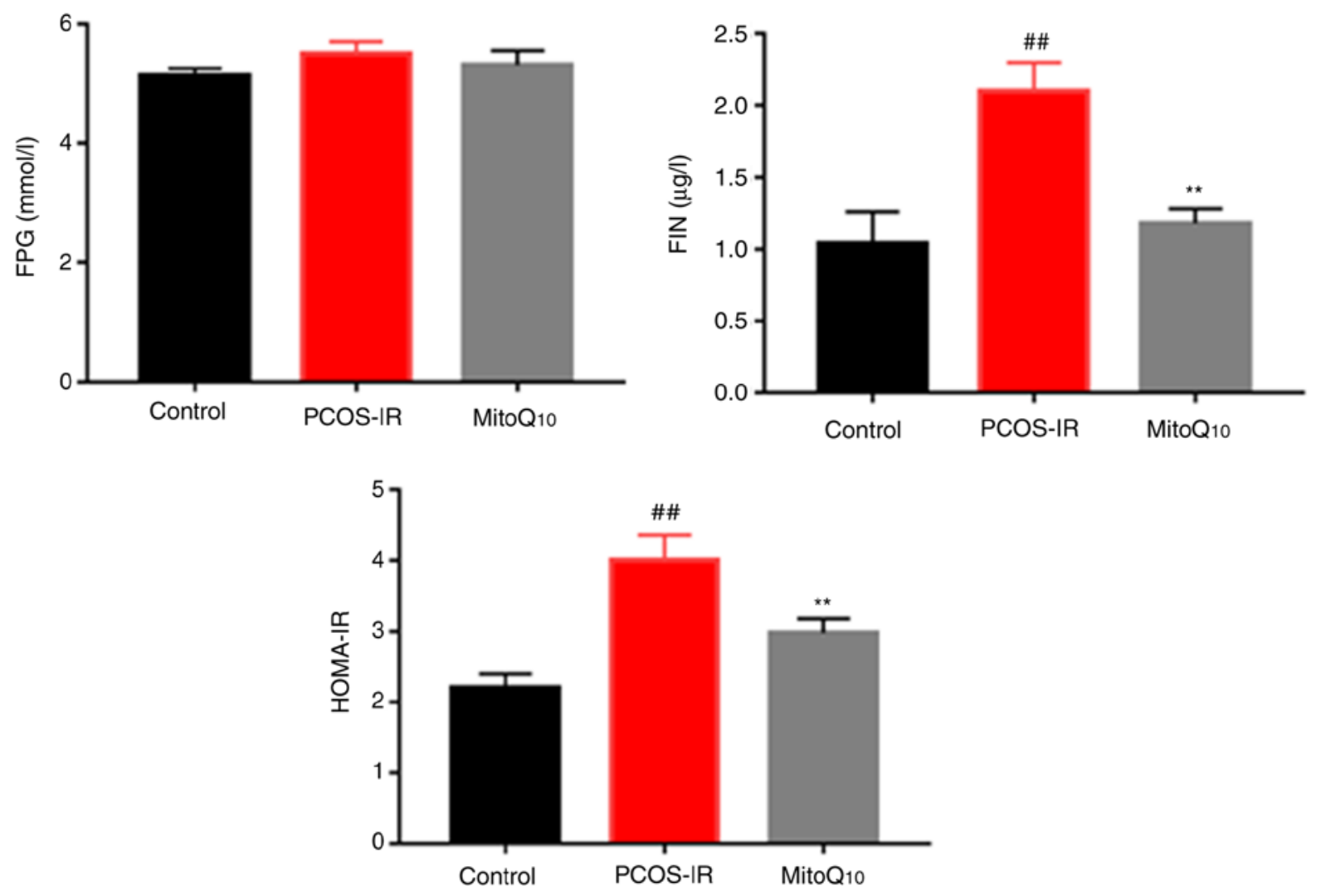

Figure 4. Serum concentrations of FPG, FIN and the HOMA-IR in the Control, PCOS-IR and $\mathrm{Mito}_{10}$ treatment groups. ${ }^{\sharp \#} \mathrm{P}<0.05$, compared with the control; ${ }^{* *} \mathrm{P}<0.05$, compared with the PCOS-IR group. PCOS-IR, polycystic ovary syndrome and insulin resistance; FPG, fasting plasma glucose; FIN, fasting insulin; HOMA-IR.

NY, USA). The results were analyzed with one-way analysis of variance followed by Tukey's post hoc test for multiple comparisons. $\mathrm{P}<0.05$ was considered to indicate a statistically significant difference.

\section{Results}

Reproductive and metabolic features of the PCOS-IR rat model. Following 8 consecutive weeks under treatment with testosterone propionate, together with a high fat diet, the PCOS-IR mouse model was successfully established. As presented in Fig. 2, the PCOS-IR animals exhibited vaginal keratosis and abnormal estrous cycle. In addition, on measuring endocrine parameters, it was noted that all animals exhibited IR as the HOMA-IR was $>2.8$.

Recovery of endocrine status following $M$ ito $Q_{10}$ treatment. As presented in Figs. 3 and 4, the rats exposed to testosterone propionate had significantly increased T, LH, LH/FSH, FIN and HOMA-IR levels, compared with those in the control group (all $\mathrm{P}<0.01$ ). Following $\mathrm{Mito}_{10}$ treatment, the levels of these parameters were decreased significantly, compared with those in the controls (all $\mathrm{P}<0.01$ ). However, no statistically significant differences were observed in the levels of FPG or FSH prior to and following $\mathrm{Mito}_{10}$ treatment.

Mito $Q_{10}$ decreases $O S$. To determine whether $\mathrm{Mito}_{10}$ reversed the OS of PCOS-IR rats, the expression levels of MDA, T-AOC, SOD and GSH were analyzed in the rat tissues. As presented in Fig. 5, the levels of T-AOC, SOD and GSH, which reflected the antioxidant capacity in rats, were enhanced in the $\mathrm{Mito}_{10}$ treatment group, compared with those in the PCOS-IR rat model (all $\mathrm{P}<0.01$ ). Consistent with this finding, the level of MDA, an OS-related biomarker, was significantly decreased following $\mathrm{Mito}_{10}$ administration $(\mathrm{P}<0.01)$.

Mito $Q_{10}$ protects against mitochondrial damage. To determine whether the administration of $M_{10} Q_{10}$ improved the mitochondrial function, the levels of ATP, MMP and ROS were analyzed in the Control, PCOS-IR and $\mathrm{MitoQ}_{10}$ treatment groups. As shown in Fig. 6, it was found that the antioxidant significantly protected against mitochondrial damage (all $\mathrm{P}<0.05)$.

Detection of the expression of Cyto C, Bcl-xL, Bcl-2 and Bax. The present study further evaluated the expression levels of the apoptotic and anti-apoptotic proteins, Cyto C, Bcl-xL, $\mathrm{Bcl}-2$ and $\mathrm{Bax}$, in the different groups to examine whether the $\mathrm{Mito}_{10}$ had a therapeutic effect. As presented in Figs. 7 and 8, it was found that, compared with the control group, the PCOS-IR rats exhibited higher levels of Cyto $\mathrm{C}$ and Bax and a higher ratio of $\mathrm{Bax}$ to $\mathrm{Bcl}-2$, whereas the level of $\mathrm{Bcl}-\mathrm{xL}$ was decreased. However, following $\mathrm{Mito}_{10}$ treatment, the levels of Cyto C, Bax and the ratio of Bax to Bcl-2 were decreased and the level of $\mathrm{Bcl}-\mathrm{xL}$ was increased, compared with levels in the PCOS-IR group. However, no statistically significant difference was observed in the level of Bcl-2 between these groups. 

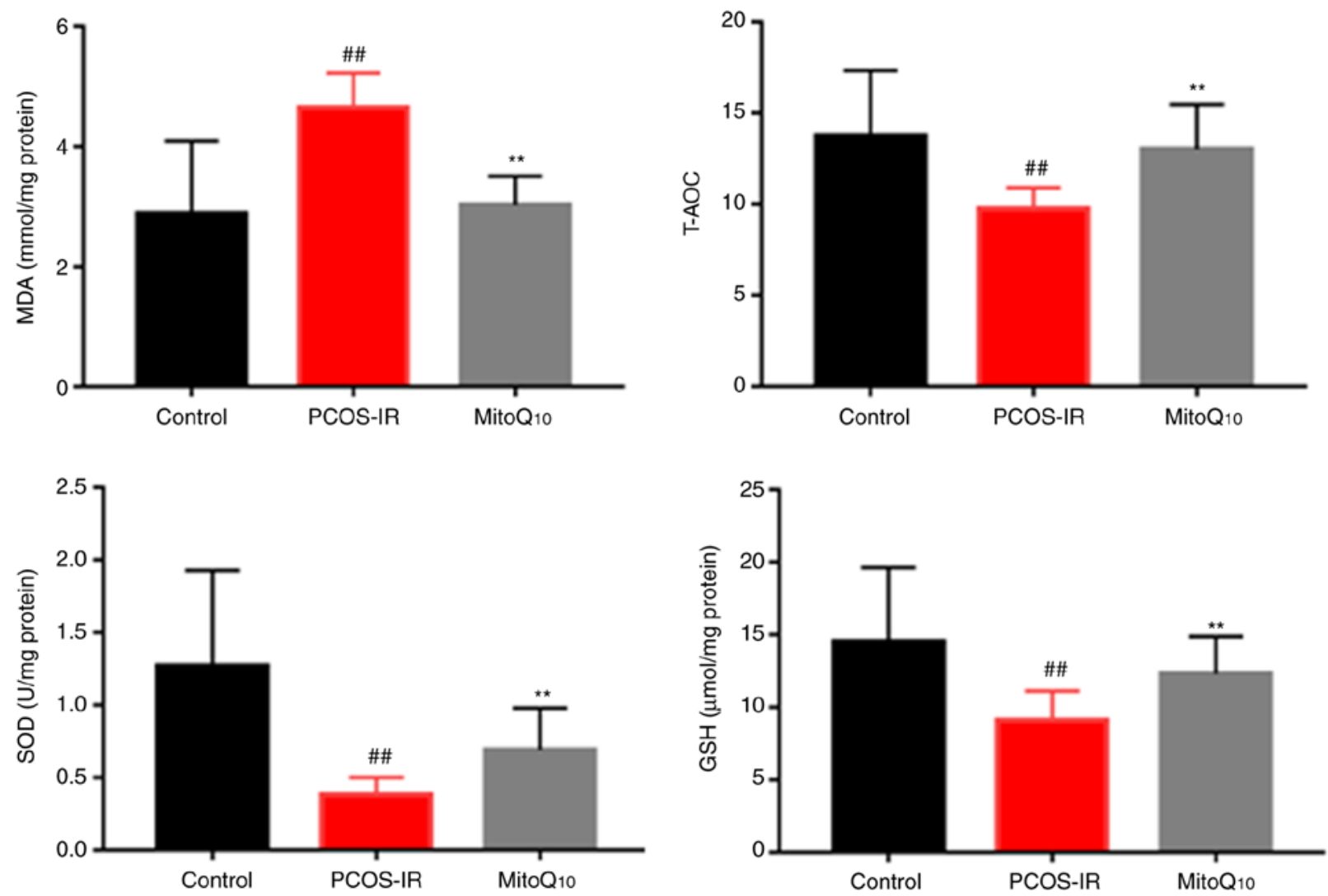

Figure 5. Analysis of oxidative stress-associated biomarkers in the Control, PCOS-IR and $\mathrm{Mito}_{10}$ treatment groups. ${ }^{\# \#} \mathrm{P}<0.05$, compared with the control; ${ }^{* *} \mathrm{P}<0.05$, compared with the PCOS-IR group. PCOS-IR, polycystic ovary syndrome and insulin resistance; MDA, malondialdehyde; T-AOC, total antioxidant capacity; SOD, superoxide dismutase; GSH, glutathione.

\section{Discussion}

In the present study, the therapeutic effects of mitochondria-targeted antioxidant $\mathrm{Mito}_{10}$ on an animal model of PCOS-IR were investigated. Accumulating evidence has demonstrated that the elevated production of ROS has an active role in the progression and pathogenesis of PCOS $(23,24)$. Natural antioxidants, including vitamin $\mathrm{C}$ and vitamin $\mathrm{E}$, cannot scavenge sufficient ROS as they cannot direct bind to mitochondria, highlighting the importance of developing novel targeted therapies. Mito $\mathrm{Q}_{10}$ can solve this problem; this antioxidant is composed of ubiquinone moieties attached to a TPP moiety by a 10-carbon alkyl chain. Lipophilic cations can readily move through phospholipid bilayers, therefore, Mito $_{10}$ can be taken up by mitochondria and be effective in combating ROS $(25,26)$. Once within the mitochondria, almost all of the accumulated $\mathrm{Mito}_{10}$ resides in the matrix surface of the inner-membrane and prevents OS and mitochondrial dysfunction (27).

In the present study, to examine the therapeutic effects of $\mathrm{Mito}_{10}$, an animal model of PCOS-IR was generated via the subcutaneous injection of testosterone propionate, together with a high-fat diet. In general, this animal model exhibits the clinical and biochemical characterizations of women with PCOS, including abnormal estrous cycles, markedly increased total body weight, polycystic ovaries and variable levels of T, LH, FSH, FIN and FPG in the serum of rats. Of the 20 rats injected, 100\% exhibited the IR features. Following treatment with $\mathrm{MitoQ}_{10}$, the morphological characteristic of polycystic ovaries and the plasma concentrations of FIN in the PCOS group were improved. These data suggested that Mito $_{10}$ exerted a therapeutic effect against the development of PCOS-IR.

MDA, T-AOC, SOD and GSH are regarded as common markers to evaluate OS levels. Of these, MDA is the product of lipid oxidation, whereas T-AOC indicates the ability of all antioxidants in different foods to clean harmful free radicals in blood and cells (28). SOD is an enzyme which can catalyze superoxide radicals into ordinary molecular oxygen or hydrogen peroxide, and GSH is another important antioxidant that can protect cells against ROS. As presented in Fig. 5, compared with those in the control group, the rats in the PCOS-IR group had higher MDA levels and lower T-AOC, SOD and GSH levels, suggesting increased OS in PCOS-IR. However, the animals treated with $\mathrm{Mito}_{10}$ exhibited decreased OS (all $\mathrm{P}<0.05$ ).

Increasing evidence suggests that the overproduction of ROS may activate stress signals to the mitochondria and be important in a number of biological processes; this suggests that mitochondrial dysfunction, together with the impaired antioxidant system, may be involved in the pathophysiological mechanism and progression of PCOS-IR $(29,30)$. There are two general pathways of apoptosis based on different apoptotic stimuli, termed the death receptor pathway and the mitochondrial-mediated pathway. In particular, increased ROS production destroys mitochondrial function, which may 

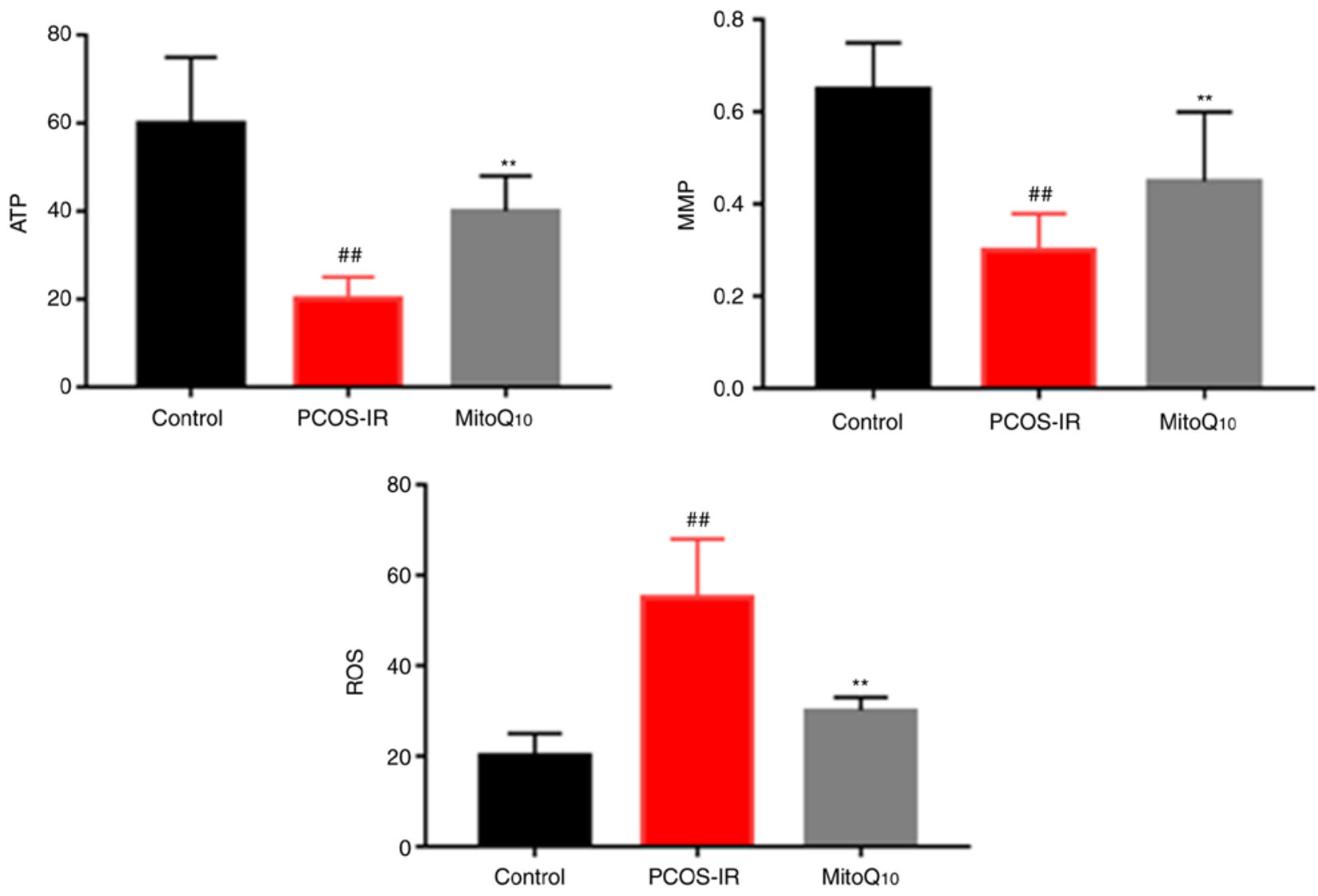

Figure 6. Analysis of mitochondrial function in the Control, PCOS-IR and Mito $\mathrm{Q}_{10}$ treatment groups. ${ }^{\# \#} \mathrm{P}<0.05$, compared with the controls; ${ }^{* *} \mathrm{P}<0.05$, compared with the PCOS-IR group. PCOS-IR, polycystic ovary syndrome and insulin resistance; MMP, mitochondrial membrane potential; ROS, reactive oxygen species.

subsequently lead to the loss of MMP and release of Cyto C $(31,32)$. Therefore, the present study measured the ROS levels, the level of MMP and the expression of apoptosis-related proteins to confirm whether $\mathrm{Mito}_{10}$ induced apoptosis via the mitochondrial pathway. The data revealed that the PCOS-IR rats treated with $\mathrm{Mito}_{10}$ exhibited recovery of mitochondrial functions, including increased ATP and MMP levels; by contrast, the ROS level was significantly decreased. This supported the hypothesis that $\mathrm{MitoQ}_{10}$ can reduce the cellular OS resulting from the mitochondrial dysfunction responsible for PCOS-IR.

$\mathrm{Bcl}-2$ and $\mathrm{Bcl}-\mathrm{xL}$ are anti-apoptotic members of the Bcl-2 family, which function as a 'life/death switch' that integrates diverse inter- and intracellular cues to determine whether or not the stress apoptotic pathway is activated $(33,34)$. In response to apoptotic stimuli, the balance and interactions of anti-apoptotic and pro-apoptotic $\mathrm{Bcl}$ proteins influence the activation of downstream pro-apoptotic proteins $\mathrm{Bcl}-2$ homologous antagonist/killer (Bak) and Bax (35). Of note, Bak and Bax are two nuclear-encoded proteins present in higher eukaryotes that are able to pierce the mitochondrial outer membrane to mediate cell death by apoptosis (36). Once the mitochondrial-mediated cell death pathway is activated, the conformations of Bax and Bak are altered, MMP is decreased and pro-apoptotic proteins are released from the intermembrane space into the cytosol $(37,38)$. Following release into the cytoplasm, Cyt C stimulates apoptosome formation followed by the activation of caspase-9. Therefore, the release of Cyt $\mathrm{C}$ is an early event in the pathway of mitochondrial-mediated
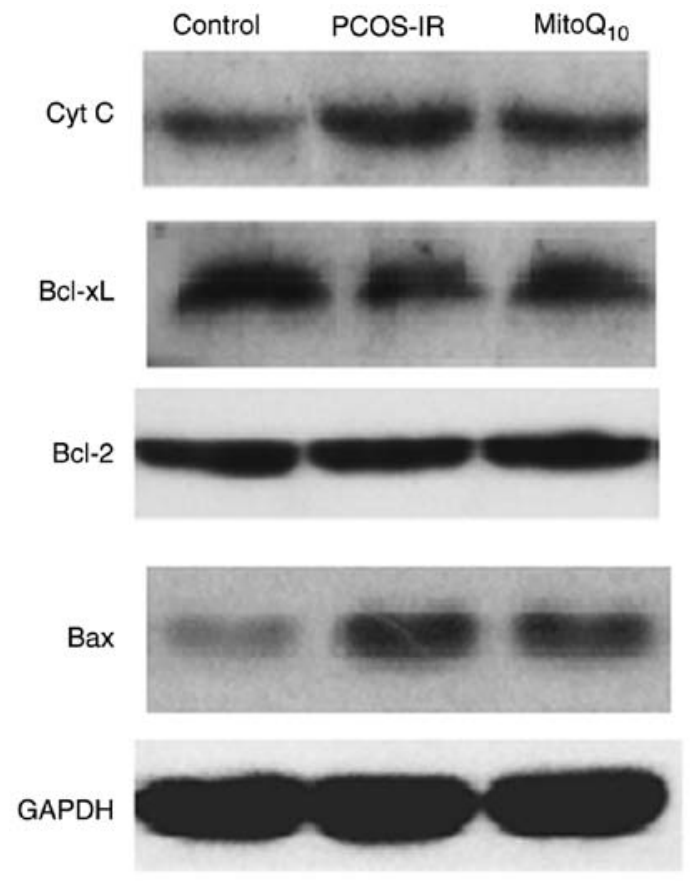

Figure 7. Relative expression of Cyt C, Bcl-xL, Bcl-2 and Bax in the ovaries of the Control, PCOS-IR and $\mathrm{Mito}_{10}$ treatment groups. PCOS-IR, polycystic ovary syndrome and insulin resistance; Cyt C, cytochrome $c$; Bcl-2, B-cell lymphoma 2; Bcl-xL, Bcl-extra large; Bax, Bcl-2-associated $\mathrm{X}$ protein.

apoptosis. As presented in Figs. 7 and 8, the present study found that $\mathrm{Mito}_{10}$ significantly increased the expression of Bcl-xL, 

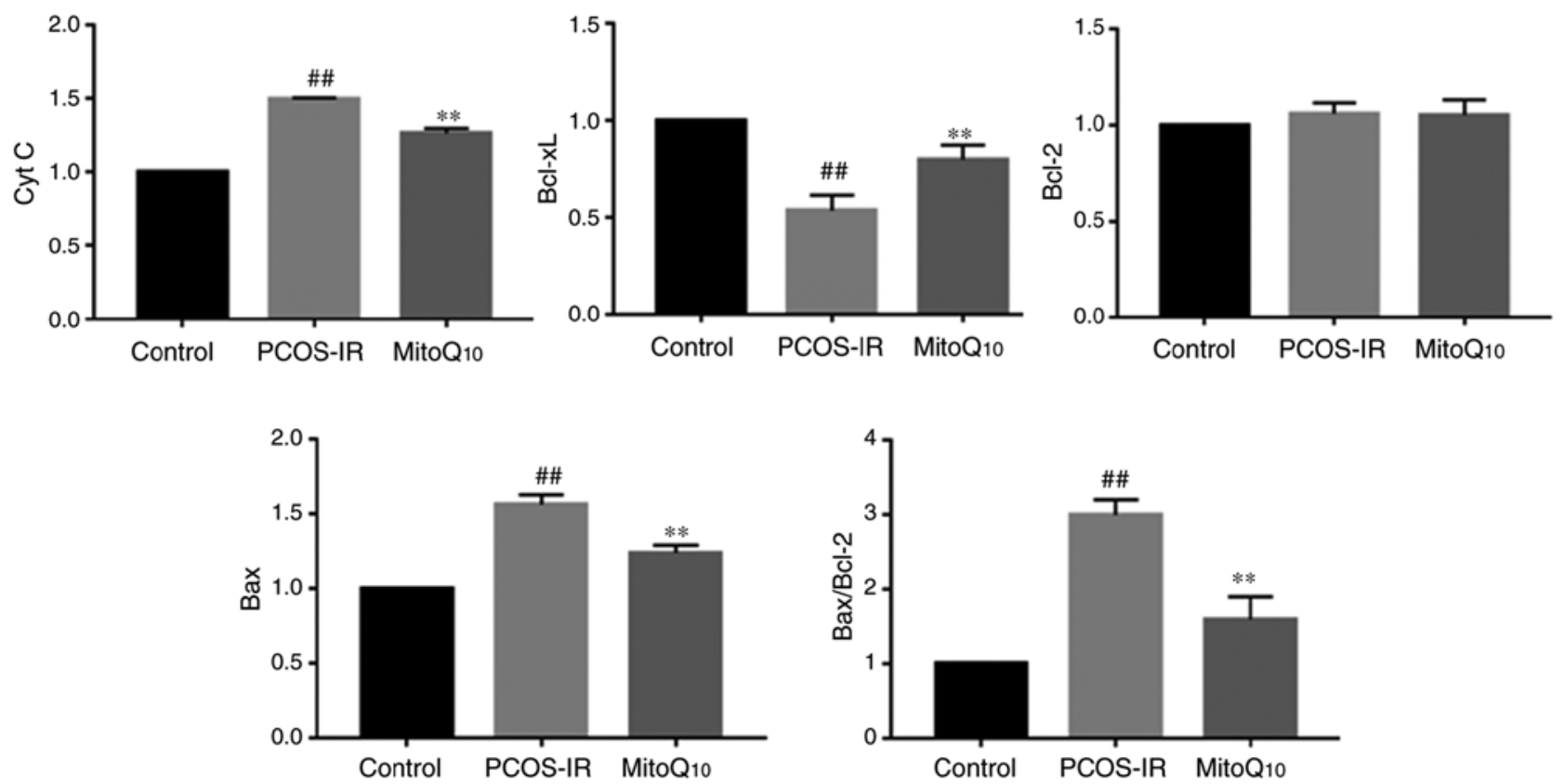

Figure 8. Qualification of the expression levels of Cyt C, Bcl-xL, Bcl-2 and Bax in the ovaries of the Control, PCOS-IR and Mito $\mathrm{Q}_{10}$ treatment groups. ${ }^{\# \#} \mathrm{P}<0.05$, compared with the control; * ${ }^{* *}<0.05$, compared with the PCOS-IR group. PCOS-IR, polycystic ovary syndrome and insulin resistance; Cyt C, cytochrome $c$; Bcl-2, B-cell lymphoma 2; Bcl-xL, Bcl-extra large; Bax, Bcl-2-associated X protein.

whereas the levels of total Cyt $\mathrm{C}$ and Bax decreased following Mito $Q_{10}$ treatment. However, no difference was observed in the expression level of $\mathrm{Bcl}-2$ prior to and following $\mathrm{Mito}_{10}$ administration, suggesting that $\mathrm{Mito}_{10}$ may not affect the downregulation of Bcl-2. A range of stimuli induce apoptosis by releasing $\mathrm{Cyt} \mathrm{C}$ from the mitochondria into the cytoplasm, where it activates caspases; the mechanisms by which these stimuli cause Cyt $\mathrm{C}$ release from mitochondria remain to be fully elucidated, however, some or all may involve increased mitochondrial OS (39). $\mathrm{Mito}_{10}$ can assist in elucidating the role of oxidative damage in apoptosis, subsequently preventing apoptotic cell death.

Although the detection of $\mathrm{Cyt} \mathrm{C}$ was not based on the total protein, it was noted that detecting total $\mathrm{Cyt} C$ protein has been reported in several studies; for example, Eleftheriadis et al (40) demonstrated that serum Cyt $\mathrm{C}$ levels may be a biomarker for mitochondrial and cellular damage. In addition, Li et al (41) measured Cyt $\mathrm{C}$ levels via an optical microfiber, and a study by Radhakrishnan et al (42) used an ELISA approach to determine plasma Cyt $\mathrm{C}$ levels. Overall, although the qualification of total Cyt $\mathrm{C}$ levels cannot reflect programmed cell death, it is only the way for reflecting the early event of apoptosis.

Based on the above observations, the present study hypothesized that the possible molecular mechanism underlying Mito $Q_{10}$ in the treatment of PCOS-IR rats may be as follows: Administration of $\mathrm{Mito}_{10}$ may bind to mitochondria and scavenge ROS, indicating its antioxidant effects. Subsequently, decreased ROS levels protect the pancreatic cells from death, most likely from mitochondrial-mediated apoptosis in the form of increasing the expression levels of Bcl-xL, decreasing the expression levels of Cyt $\mathrm{C}$ and $\mathrm{Bax}$, and recovering mitochondrial functions, with improved ATP and MMP levels. As a result, the decreased OS may prevent the pancreatic cells from death or apoptosis. Finally, the endocrine and reproductive conditions of PCOS-IR recovered following $\mathrm{Mito}_{10}$ treatment. Therefore, this antioxidant may be a potential agent for patients with PCOS-IR in the future.

\section{Acknowledgements}

The authors would like to thank the members of their lab for discussion. The authors are grateful to Dr Qi Liu from Shaoxing People's Hospital, Zhejiang University for critical reading of this manuscript.

\section{Funding}

This study was supported by the grants from the Hangzhou Bureau of Science and Technology (grant no. 20150633B16), the Ministry of Public Health from Zhejiang Province (grant nos. 2013KYA158 and 2018ZH019) and the Natural Science Foundation of Zhejiang Province (grant nos. LY14H270008 and LY15H280007).

\section{Availability of data and materials}

The datasets used and/or analyzed during the present study are available from the corresponding author on reasonable request.

\section{Authors' contributions}

YD designed the experiments, ZJ performed the histopathological analysis, BX performed the statistical analysis, LZ performed the animal experiments, $\mathrm{CZ}$ and JL analyzed the data. YD wrote the manuscript. All authors discussed the results and implications and commented on the manuscript at all stages. All authors read and approved the final manuscript. 


\section{Ethics approval and consent to participate}

All experiments were approved by the Animal Care and Use Committee of Zhejiang Chinese Medical University.

\section{Patient consent for publication}

Not applicable.

\section{Competing interests}

The authors declare that they have no competing interests.

\section{References}

1. McCartney CR and Marshall JC: Clinical Practice. Clinical practice. Polycystic ovary syndrome. N Engl J Med 375: 54-64, 2016.

2. Ali AT: Polycystic ovary syndrome and metabolic syndrome. Ceska Gynekol 80: 279-289, 2015.

3. Kalyanaraman B: Teaching the basics of redox biology to medical and graduate students: Oxidants, antioxidants and disease mechanisms. Redox Biol 1: 244-257, 2013.

4. Liu X, Zhu Q, Zhang M, Yin T, Xu R, Xiao W, Wu J, Deng B, Gao X, Gong W, et al: Isoliquiritigenin ameliorates acute pancreatitis in mice via inhibition of oxidative stress and modulation of the Nrf2/HO-1 pathway. Oxid Med Cell Longev 2018: 7161592, 2018.

5. Dalle-Donne I, Rossi R, Colombo R, Giustarini D and Milzani A: Biomarkers of oxidative damage in human disease. Clin Chem 52: 601-623, 2006.

6. Escobar-Morreale HF, Luque-Ramírez M and San Millán JL: The molecular-genetic basis of functional hyperandrogenism and the polycystic ovary syndrome. Endocr Rev 26: 251-282, 2005.

7. Zuo T, Zhu M and Xu W: Roles of oxidative stress in polycystic ovary syndrome and cancers. Oxid Med Cell Longev 2016: 8589318, 2016

8. Picard M, Wallace DC and Burelle Y: The rise of mitochondria in medicine. Mitochondrion 30: 105-116, 2016.

9. Böttger EC and Schacht J: The mitochondrion: A perpetrator of acquired hearing loss. Hear Res 303: 12-19, 2013.

10. Ding Y, Xia BH, Zhang CJ and Zhuo GC: Mitochondrial tRNALeu(UUR) C3275T, tRNAGln T4363C and tRNALys A8343G mutations may be associated with PCOS and metabolic syndrome. Gene 642: 299-306, 2018.

11. Ding Y, Xia BH, Zhang CJ and Zhuo GC: Mutations in mitochondrial tRNA genes may be related to insulin resistance in women with polycystic ovary syndrome. Am J Transl Res 9: 2984-2996, 2017.

12. Kelso GF, Porteous CM, Coulter CV, Hughes G, Porteous WK, Ledgerwood EC, Smith RA and Murphy MP: Selective targeting of a redox-active ubiquinone to mitochondria within cells: antioxidant and antiapoptotic properties. J Biol Chem 276: 4588-4596, 2001.

13. Smith RA and Murphy MP: Animal and human studies with the mitochondria-targeted antioxidant MitoQ. Ann NY Acad Sci 1201: 96-103, 2010

14. ManczakM,MaoP,CalkinsMJ,CorneaA,Reddy AP,MurphyMP, Szeto HH, Park B and Reddy PH: Mitochondria-targeted antioxidants protect against amyloid-beta toxicity in Alzheimer's disease neurons. J Alzheimers Dis 20 (Suppl 2): S609-S631, 2010.

15. Mao P, Manczak M, Shirendeb UP and Reddy PH: MitoQ, a mitochondria-targeted antioxidant, delays disease progression and alleviates pathogenesis in an experimental autoimmune encephalomyelitis mouse model of multiple sclerosis. Biochim Biophys Acta 1832: 2322-2331, 2013.

16. Graham D, Huynh NN, Hamilton CA, Beattie E, Smith RA, Cochemé HM, Murphy MP and Dominiczak AF: Mitochondria-targeted antioxidant MitoQ10 improves endothelia function and attenuates cardiac hypertrophy. Hypertension 54: 322-328, 2009
17. McLachlan J, Beattie E, Murphy MP, Koh-Tan CH, Olson E, Beattie W, Dominiczak AF, Nicklin SA and Graham D: Combined therapeutic benefit of mitochondria-targeted antioxidant, MitoQ10, and angiotensin receptor blocker, losartan, on cardiovascular function. J Hypertens 32: 555-564, 2014.

18. Keskin M, Kurtoglu S, Kendirci M, Atabek ME and Yazici C: Homeostasis model assessment is more reliable than the fasting glucose/insulin ratio and quantitative insulin sensitivity check index for assessing insulin resistance among obese children and adolescents. Pediatrics 115: e500-e503, 2005.

19. Yarian CS, Toroser D and Sohal RS: Aconitase is the main functional target of aging in the citric acid cycle of kidney mitochondria from mice. Mech Ageing Dev 127: 79-84, 2006.

20. Yuan Y, Chen Y, Zhang P, Huang S, Zhu C, Ding G, Liu B, Yang $T$ and Zhang A: Mitochondrial dysfunction accounts for aldosterone-induced epithelial-to-mesenchymal transition of renal proximal tubular epithelial cells. Free Radic Biol Med 53: 30-43, 2012.

21. Raza H, John A and Howarth FC: Increased oxidative stress and mitochondrial dysfunction in zucker diabetic rat liver and brain. Cell Physiol Biochem 35:1241-1251, 2015.

22. Dignam JD, Lebovitz RM and Roeder RG: Accurate transcription initiation by RNA polymerase II in a soluble extract from isolated mammalian nuclei. Nucleic Acids Res 11: 1475-1489, 1983.

23. Victor VM, Rovira-Llopis S, Bañuls C, Diaz-Morales N, Martinez de Marañon A, Rios-Navarro C, Alvarez A, Gomez M, Rocha M and Hernández-Mijares A: Insulin resistance in PCOS patients enhances oxidative stress and leukocyte adhesion: Role of myeloperoxidase. PLoS One 11: e0151960, 2016.

24. Serrano Mujica L, Bridi A, Della Méa R, Rissi VB, Guarda N, Moresco RN, Premaor MO, Antoniazzi AQ, Gonçalves PBD and Comim FV: Oxidative stress and metabolic markers in pre- and postnatal polycystic ovary syndrome rat protocols. J Inflamm Res 11: 193-202, 2018.

25. Ross MF, Kelso GF, Blaikie FH, James AM, Cochemé HM, Filipovska A, Da Ros T, Hurd TR, Smith RA and Murphy MP: Lipophilic triphenylphosphonium cations as tools in mitochondrial bioenergetics and free radical biology. Biochemistry (Mosc) 70: 222-230, 2005.

26. Firsov AM, Kotova EA, Orlov VN, Antonenko YN and Skulachev VP: A mitochondria-targeted antioxidant can inhibit peroxidase activity of cytochrome $\mathrm{c}$ by detachment of the protein from liposomes. FEBS Lett 590: 2836-2843, 2016.

27. James AM,CocheméHMand Murphy MP:Mitochondria-targeted redox probes as tools in the study of oxidative damage and ageing. Mech Ageing Dev 126: 982-986, 2005.

28. Yilmaz N, Inal HA, Gorkem U, Sargin Oruc A, Yilmaz S and Turkkani A: Follicular fluid total antioxidant capacity levels in PCOS. J Obstet Gynaecol 36: 654-657, 2016.

29. Abu Bakar MH, Sarmidi MR, Tan JS and Mohamad Rosdi MN: Celastrol attenuates mitochondrial dysfunction and inflammation in palmitate-mediated insulin resistance in C3A hepatocytes. Eur J Pharmacol 799: 73-83, 2017.

30. Hafizi Abu Bakar M, Kian Kai C, Wan Hassan WN, Sarmidi MR, Yaakob $\mathrm{H}$ and Zaman Huri H: Mitochondrial dysfunction as a central event for mechanisms underlying insulin resistance: The roles of long chain fatty acids. Diabetes Metab Res Rev 31: 453-475, 2015.

31. Yan Y, Su X, Liang Y, Zhang J, Shi C, Lu Y, Gu L and Fu L: Emodin azide methyl anthraquinone derivative triggers mitochondrial-dependent cell apoptosis involving in caspase-8-mediated Bid cleavage. Mol Cancer Ther 7: 1688-1697, 2008.

32. Wang XH, Jia DZ, Liang YJ, Yan SL, Ding Y, Chen LM, Shi Z, Zeng MS, Liu GF and Fu LW: Lgf-YL-9 induces apoptosis in human epidermoid carcinoma KB cells and multidrug resistant KBv200 cells via reactive oxygen species-independent mitochondrial pathway. Cancer Lett 249: 256-270, 2007.

33. Kluck RM, Bossy-Wetzel E, Green DR and Newmeyer DD: The release of cytochrome $\mathrm{c}$ from mitochondria: a primary site for Bcl-2 regulation of apoptosis. Science 275: 1132-1136, 1997.

34. Yang J, Liu X, Bhalla K, Kim CN, Ibrado AM, Cai J, Peng TI, Jones DP and Wang X: Prevention of apoptosis by Bcl-2: Release of cytochrome c from mitochondria blocked. Science 275: 1129-1132, 1997.

35. Nechushtan A, Smith CL, Lamensdorf I, Yoon SH and Youle RJ: Bax and Bak coalesce into novel mitochondria-associated clusters during apoptosis. J Cell Biol 153: 1265-1276, 2001.

36. Susin SA, Lorenzo HK, Zamzami N, Marzo I, Snow BE, Brothers GM, Mangion J, Jacotot E, Costantini P, Loeffler M, et al: Molecular characterization of mitochondrial apoptosis-inducing factor. Nature 397: 441-446, 1999. 
37. Zhang JY, Yi T, Liu J, Zhao ZZ and Chen HB: Quercetin induces apoptosis via the mitochondrial pathway in KB and KBv200 cells. J Agric Food Chem 61: 2188-2195, 2013.

38. Westphal D, Dewson G, Czabotar PE and Kluck RM: Molecular biology of Bax and Bak activation and action. Biochim Biophys Acta 1813: 521-531, 2011.

39. Hampton MB and Orrenius S: Dual regulation of caspase activity by hydrogen peroxide: Implications for apoptosis. FEBS Lett 414: 552-556, 1997.

40. Eleftheriadis T, Pissas G, Liakopoulos V and Stefanidis I: Cytochrome c as a potentially clinical useful marker of mitochondrial and cellular damage. Front Immunol 7: 279, 2016.

41. Li H, Huang Y, Chen C, Xiao A, Hou G, Huang Y, Feng X and Guan BO: Real-time cellular cytochrome c monitoring through an optical microfiber: Enabled by a silver-decorated graphene nanointerface. Adv Sci (Weinh) 5: 1701074, 2018.
42. Radhakrishnan J, Origenes R, Littlejohn G, Nikolich S, Choi E, Smite S, Lamoureux L, Baetiong A, Shah M and Gazmuri RJ: Plasma cytochrome $\mathrm{c}$ detection using a highly sensitive electrochemiluminescence enzyme-linked immunosorbent assay. Biomark Insights 12: 1177271917746972, 2017.

(i) $(9)$ This work is licensed under a Creative Commons Attribution-NonCommercial-NoDerivatives 4.0 International (CC BY-NC-ND 4.0) License. 